\title{
Approbation définitive des directives concernant les «Directives anticipées»
}

Peter Lack, Michelle Salathé

\begin{abstract}
Le Sénat de I'ASSM a approuvé les directives concernant les «Directives anticipées» le 19 mai 2009. La version définitive peut être téléchargée sur le site de I'ASSM (www.assm.ch) ou commandée sous forme de brochure au secrétariat général de I'ASSM, Petersplatz 13, 4051 Bâle.
\end{abstract}

Correspondance:

Michelle Salathé

Secrétariat général de l'ASSM

Petersplatz 13

CH-4051 Bâle
En novembre 2008, l'Académie Suisse des Sciences Médicales (ASSM) avait mis en consultation un projet de directives concernant la rédaction de directives anticipées et leur application dans la pratique médicale quotidienne. Les nombreuses prises de position (environ 80), majoritairement positives, parvenues au secrétariat général de l'ASSM pendant la période de consultation témoignent de l'intérêt pour ce projet de directives. Les directives ont été considérées comme une «aide précieuse et pratique pour l'élaboration de directives anticipées». Les réponses critiques concernaient principalement l'importance à accorder au conseil lors de la rédaction de directives anticipées ainsi que le caractère contraignant des directives anticipées. Egalement des questions connues et déjà discutées au sein de la sous-commission ont été soulevées: alors que les uns souhaitent des directives anticipées aussi simples et «rapides» que possible, les autres signalent justement les problèmes liés à de telles directives au moment de leur application. Par ailleurs, il a été souhaité que la liste des destinataires de ces directives soit élargie à des personnes individuelles impliquées et aux organisations de conseil.

Lors de la rédaction finale du texte, la sous-commission, dirigée par lic. théol. Peter Lack de Bâle, a tenu compte de la plupart de ces propositions. Concernant le caractère contraignant des directives anticipées, les directives de l'ASSM s'orientent vers la révision du Code Civil (protection de l'adulte) qui entrera en vigueur en 2012 au plus tôt. Jusque là, les réglementations cantonales existantes sont valables. Toutefois, celles-ci divergent d'un canton à l'autre et certains cantons n'ont pas de règlement explicite. En principe, la règle suivante est actuellement appliquée: plus les directives anticipées sont claires et plus elles anticipent la situation de façon concrète, plus elles auront d'importance lors de la prise de décision.

\section{Contenus des directives anticipées}

Les directives anticipées permettent à une personne de consigner par écrit à quelles mesures médicales elle consentirait et celles qu'elle refuserait en cas d'incapacité de discernement. Elles peuvent aussi contenir des indications concernant d'autres sujets, comme par exemple l'autopsie, la transplantation, etc. et/ou désigner un représentant thérapeutique. Il est souvent difficile de définir à l'avance les mesures médicales indiquées dans une situation concrète, c'est pourquoi, il est conseillé à l'auteur de décrire son échelle personnelle de valeurs. De telles indications peuvent donner des indices précieux lorsque l'auteur ne s'est pas exprimé de façon explicite sur des situations ou des mesures spécifiques ou lorsqu'il n'est pas possible de prévoir l'issue positive d'un traitement médical.

Les directives anticipées ne peuvent pas contenir des exigences contraires à la Loi. De même, des traitements qui ne sont pas médicalement indiqués ne peuvent pas être exigés. Par contre, des traitements peuvent être refusés, même s'ils sont médicalement indiqués. Dans ce cas, il est recommandé de faire mention des motifs, afin de ne laisser subsister aucun doute sur ce qui constitue la volonté du patient au moment d'appliquer les directives.

\section{Le recours à un conseiller n'est pas obligatoire, mais vivement recommandé}

Les directives précisent expressément qu'il n'y a aucune obligation de conseil lors de la rédaction de directives anticipées; toutefois, selon l'ASSM un conseiller peut s'avérer précieux et est vivement recommandé. Le médecin de famille, le médecin spécialiste ou d'autres professionnels compétents et expérimentés peuvent se charger de la séance de conseil. Les points essentiels d'une séance de conseil incluent la réflexion et la documentation relatives à l'échelle personnelle des valeurs, l'information concernant les situations possibles d'incapacité de discernement ainsi que celle relative aux mesures médicales habituelles dans ces situations.

\section{Application des directives anticipées}

Les directives abordent aussi les situations dans lesquelles des directives anticipées ne correspondent plus à la volonté du patient, sans que ce dernier les ait formellement révoqué ou modifié. Sont également décrites des situations où les avis des médecins traitants, des soignants, des représentants thérapeutiques et des proches divergent quant à l'interprétation des directives anticipées. Face à une telle situation, les directives décrivent les processus décisionnels et les mesures possibles de soutien.

Afin de tenir compte des souhaits exprimés à maintes reprises pendant la période de consultation et de renforcer l'impact des directives anticipées dans une situation concrète, l'ASSM va élaborer, d'une part, une version résumée de ces directives et, d'autre part, des questions types pour la description de l'échelle des valeurs à laquelle l'ASSM accorde beaucoup d'importance. 